\title{
Nutrition and sarcopenia in posmenopausal women
}

\section{DOI: $\underline{\text { http://doi.org/10.26758/7.1.11 }}$}

Andrei Kozma (1), Sasa Pocnetz (2), Gabriel-Ioan Prada (3)

(1) National Institute for Mother and Child Health "Alessandrescu Rusescu", Bucharest, Romania

(2) University Hospitals Birmingham, Birmingham, United Kingdom

(3)"Ana Aslan" National Institute of Gerontology and Geriatrics, Bucharest, Romania

Address correspondence to: Dr.Andrei Kozma, "Fr.I.Rainer" Institute of Anthropology of Romanian Academy, Casa Academiei, Bd."13 Septembrie", No.13, Sector 5, Bucharest, Romania; E-mail address: dr.ka.mailbox@gmail.com

\begin{abstract}
Objectives. Sarcopenia is an important preliminary factor for frailty syndrome. The aim of the study was to evaluate the association between nutrition and other potential risk factors and sarcopenia in post-menopausal women.

Materials and Methods. A total of 352 post-menopausal patients consecutively admitted to "Ana Aslan" National Institute of Gerontology and Geriatrics have been evaluated. Patients with diseases that could interfere with their nutritional status have been excluded. Following parameters have been considered: age, age of menopause onset, time elapsed since menopause onset, level of school education, average income, area of residence, living arrangements nutritional status and mood disorders. Measuring gait speed on a 6-m course assessed the risk of physical disability and sarcopenia.
\end{abstract}

Results. A significant negative correlation ( $\mathrm{r}=-0.89$; $\mathrm{p}<0.05)$ was noticed between muscle performance and chronological age. Sarcopenia was not correlated with age of menopause onset. The risk of malnutrition was significantly associated with the risk of sarcopenia $(r=0.73 ; \mathrm{p}<0.05)$. The level of formal education did not correlate with sarcopenia, but patients having family support had a lower risk of physical disability $(\mathrm{p}<0.05)$. There was a significant negative association between the presence of mood disorders and gait speed $(\mathrm{r}=0.58 ; \mathrm{p}<0.05)$.

Conclusions. In conclusion, factors such as the presence of mood disorders and enhanced risk of malnutrition have a significant influence on the occurrence of sarcopenia in postmenopausal women.

Keywords: sarcopenia, older people, nutrition, anthropological

\section{Introduction}

Sarcopenia represents an important condition in older people consisting of a progressive decline in skeletal muscle mass and performance (Cruz-Jentoft et al., 2010). It is thought to affect $30 \%$ of people beyond the age of 60 years and its prevalence increases with age reaching more than $50 \%$ of people over 80 years (Santilli et al. 2014; Cruz-Jentoft et al., 2014; Bergamaschine Mata Diz et al, 2015; Dodds et al. 2015). Sarcopenia is accompanied by a multi-system impairment associated with increased vulnerability to a variety of risk factors (Dodds et al., 2016). There are several definitions for sarcopenia (Cawthon et al., 2015), but some studies showed that they have limited clinical utility in healthy, community-dwelling men, albeit there are no such studies in women. 
However, by any definition, sarcopenia is correlated with several adverse health outcomes especially recurrent falls and increased risk of mortality.

There is a considerable overlap between sarcopenia and frailty, the latter being a syndrome relatively recently defined as a multisystem impairment associated with increased vulnerability to stressor events (Clegg et al, 2013). The definitions of the two conditions contain some similar items: gait speed, grip strength, muscle mass, weight loss. Many times frailty is accompanied by sarcopenia, but frailty is not always limited to sarcopenia and not all frail older people present sarcopenia, especially in early stages.

Inactivity is significantly correlated with loss of muscle mass and strength. This supports the idea that physical activity could be a protective factor for the prevention and also for the management of sarcopenia. Furthermore, one of the first steps to be taken in the management of sarcopenia or clinical frailty is to ensure that the sarcopenic patient is receiving correct and sufficient nutrition. Three main areas regarding dietary interventions have been considered: proteins, antioxidants and vitamin D. However, the quantity and composition of dietary protein intake for the prevention and treatment of sarcopenia are still controversial since a Cochrane review failed to find any significant improvement in functional benefit or reduction in length of hospital stay with supplements (Millan et al., 2009). There is a general consensus that low vitamin D levels require correction, but studies on its effects on strength and physical performance gave mixed results (Dodds et al., 2016; Collamati et al., 2016). Some observational studies have shown that persons with higher antioxidant status have better physical performance and a lower decline in walking speed (Kaiser at al., 2010). Nevertheless, to date, there are no clear data regarding the precise forms of free radical species responsible for muscle wasting and which specific antioxidants are likely to be of benefit.

Sarcopenia is more prevalent in women in most countries except Taiwan where sarcopenia is more than twice more prevalent in men and in Japan where there is no significant difference between the two genders (Bergamaschine Mata Diz et al., 2015). The age-related muscle mass reduction is not linear and does not occur with the same rates in men and women (Lindle et al., 1997). Some studies have shown that the accelerated loss of skeletal muscle mass and performance occurs earlier in women than in men, during the premenopausal period (Maltais et al., 2009). The decline in estrogen levels contributes to the redistribution of body fat to the visceral area, decreased bone mineral density and potentially loss of muscle tissue (Messier et al., 2011).

Sarcopenia has a great negative effect on survival and quality of life in older people. It is important to prevent or postpone as much as possible the onset of this condition, in order to enhance survival and to reduce the demand for long-term care. The main objective of this study is to evaluate the association of various potential risk factors with sarcopenia in postmenopausal women.

\section{Materials and Methods}

A total of 352 post-menopausal female patients consecutively admitted to "Ana Aslan" National Institute of Gerontology and Geriatrics were selected based on eligibility criteria. The inclusion criterion was postmenopausal status defined as at least 12 consecutive months of amenorrhea. Potential study participants were checked against the following exclusion criteria: moderate-to-severe and severe neurocognitive deficits, end-stage chronic diseases, patients in acute phase of chronic diseases or with acute diseases. Nutritional status was assessed with the Mini Nutritional Assessment $\left(\mathrm{MNA}^{\circledR}\right)$ scale. The $\mathrm{MNA}^{\circledR}$ nutrition screening and assessment standardized instrument is a validated tool that identifies patients who are malnourished or at risk of malnutrition (Rubenstein LZ et al., 2001). It includes evaluations of seven domains: decreased nutritional uptake 
due to lack of appetite or digestive tract disorders, weight loss, mobility assessment, psychological stress or acute illness, body mass index or calf circumference and neurocognitive and mood disorders. A score of 0-7 points classifies malnutrition, 8-11 points risk of malnutrition and 12-14 points normal nutritional status (Guigoz Y., 2006; Rubenstein LZ et al., 2001). The European Working Group on Sarcopenia in Older People (EWGSOP) developed recommendations on sarcopenia risk evaluation in clinical practice (Cruz-Jentoft AJ et al., 2010). In our study, we used the gait speed as an EWGSOP endorsed measure of muscle performance. According to EWGSOP, a cut-off point of $>0.8 \mathrm{~m} / \mathrm{s}$ ascertains the risk for sarcopenia (Cruz-Jentoft AJ et al., 2010). Investigators applied the 6-meter course walking assessment to each individual patient included in the study. Data from patients' charts have been collected: age, place of residence, income (pension slip), living arrangements (alone/not alone), the level of formal education, the presence of menopause and time elapsed since its onset, a diagnosis of mood disorders.

Student's t-test was used for testing the statistical significance of the differences between nutritional status groups for gait speed. A Pearson's correlation coefficient was used to assess associations between risk of sarcopenia and age, time elapsed since the onset of menopause and sociodemographic factors. A statistical significant level was considered for $p<0,05$. Statistical analysis was performed with the DATAPLOT software system.

\section{Results}

The sociodemographic characteristics of the study group are depicted in Table 1 . The mean age was 74 years (minimum 54, maximum 92) and most patients were living in urban areas. The majority of study participants had undergone at least 12 years of formal education. Compared to the national average monthly income, $62.5 \%$ of our study participants had higher pensions. Most persons $(72.15 \%)$ were living with a spouse or other relatives. Depressive and anxiety disorders were present in $36.07 \%$ of patients.

Table 1. Sociodemographic characteristics of the study group

\begin{tabular}{|c|c|c|}
\hline & $\begin{array}{c}\text { Study group }(\mathrm{N}=352) \\
\text { Mean }(\mathrm{SD}) / \mathrm{n}(\%)\end{array}$ \\
\hline \multicolumn{2}{|l|}{ Age } & $74.08(7.21)$ \\
\hline \multicolumn{2}{|c|}{ Age at menopause onset } & $50.26(4.81)$ \\
\hline \multicolumn{2}{|c|}{ Years since menopause onset } & $23.37(2.12)$ \\
\hline \multirow[t]{2}{*}{ Residence } & rural & $112(31.81)$ \\
\hline & urban & $240(68.18)$ \\
\hline \multirow[t]{3}{*}{ Level of education } & primary school & $37(10.51)$ \\
\hline & high-school & $189(53.69)$ \\
\hline & college/university & $126(35.79)$ \\
\hline \multirow[t]{2}{*}{ Income* } & $<848$ & $132(37.5)$ \\
\hline & $\geq 848$ & $220(62.5)$ \\
\hline \multirow[t]{2}{*}{ Living arrangements } & alone & $147(41.76)$ \\
\hline & not alone & $205(58.23)$ \\
\hline \multicolumn{2}{|l|}{ Mood disorders } & $127(36.07)$ \\
\hline
\end{tabular}

*Average pension/month expressed in local currency RON. 
Nutritional status evaluated with $\mathrm{MNA}^{\circledR}$ indicated that $20.44 \%$ of participants were malnourished or at risk of malnutrition and $79.56 \%$ had a normal nutritional status at the time of the assessment (Table 2). In our sample, $19.6 \%$ of postmenopausal women had gait speeds below the cut-off point for sarcopenia (Table 2).

Table 2. Nutritional status and gait speed

\begin{tabular}{|l|c|}
\hline MNA $^{\circledR}$ Score & $\mathrm{N}(\%)$ \\
\hline Malnutrition & $19(5.39)$ \\
\hline Risk of malnutrition & $53(15.05)$ \\
\hline Normal nutritional status & $280(79.56)$ \\
\hline High risk of sarcopenia* & $69(19.6)$ \\
\hline Low risk of sarcopenia* & $283(80.4)$ \\
\hline
\end{tabular}

*Gait speed cut-off point $0.8 \mathrm{~m} / \mathrm{s}$

Based on the preliminary data, we performed multiple Student's t-test analyses to compare gate speed in patients living in urban and rural areas, patients with an income level below and above the national average, patients with low and high levels of formal education and persons living alone or with a spouse or relative. Because most patients had a normal nutritional status (79.56\%) we could not perform a meaningful statistical comparison between groups. Gait speed was significantly lower in subjects living in urban areas compared to those living in rural zones $(1.68 \mathrm{~m} / \mathrm{s} ; \mathrm{SD}=0.21)$ respectively $1.94 \mathrm{~m} / \mathrm{s}(\mathrm{SD}=0.24) ; \mathrm{p}<0.05$ ) (Table 3$)$. We recorded significantly higher gait speeds in women with above national average pensions compared to those with low income $(\mathrm{p}<0.05)$. There was a significant difference in walking speed in patients living alone compared to those living with a spouse or a relative who performed better $(\mathrm{p}<0.05)$. We found no statistical difference in walking speed test for groups with different level of education $(\mathrm{p}>0.05)$ (Table 3$)$.

Table 3. Gait speed in various interest groups

\begin{tabular}{|l|c|c|c|}
\hline Gait speed(m/s) & N/\% & Mean (SD) & p value \\
\hline Urban & $240(68.18)$ & $1.68(0.21)$ & p $=\mathbf{0 . 0 4}$ \\
\hline Rural & $112(31.81)$ & $1.94(0.24)$ & \\
\hline Low income & $132(37.5)$ & $1.47(0.37)$ & $\mathbf{p}=\mathbf{0 . 0 2}$ \\
\hline High income & $220(62.5)$ & $2.01(0.24)$ & \\
\hline $\begin{array}{l}\text { Low education level } \\
\text { (primary + high school) }\end{array}$ & $226(64.20)$ & $2.07(0.19)$ & $\mathrm{p}=0.19$ \\
\hline Higher education level & $126(35.79)$ & $2.04(0.22)$ & \\
\hline Living alone & $147(41.76)$ & $0.89(0.18)$ & $\mathbf{p}=\mathbf{0 . 0 0 8}$ \\
\hline Not loving alone & $205(58.23)$ & $2.11(0.28)$ & \\
\hline
\end{tabular}

$\mathrm{p}<0.05$ was considered statistically significant; $95 \%$ CI

Analyzing various types of factors that could influence the presence of sarcopenia, a negative significant correlation $(\mathrm{r}=-0.89 ; \mathrm{p}<0.05)$ between chronological age and muscle performance represented by gait speed has been identified. Nevertheless, the age at menopause onset did not correlate with walking speed $(\mathrm{r}=0.45 ; \mathrm{p}=0.08)$. Another factor that had a significant positive association with enhanced risk of sarcopenia was the presence of mood disorders $(r=0.58$; 
$\mathrm{p}<0.05)$. The risk of malnutrition was significantly associated with the risk of sarcopenia $(\mathrm{r}=0.73$; $\mathrm{p}<0.05)$.

\section{Discussion}

Recently, sarcopenia and its most important consequence frailty syndrome have gained an increasing attention. This was mainly due to an interesting correlation between sarcopenia and frailty syndrome, on one side, and vulnerability to various risk factors, on the other side. In our sample of postmenopausal women, the prevalence of enhanced risk of sarcopenia was $19.6 \%$. Walking speeds below the threshold for sarcopenia risk were more frequent among postmenopausal women in other reports (McGinn AP et al., 2008; Bohannon RW et al., 2011; Park H et al., 2013). The enhanced risk of sarcopenia was positively associated with older age. Nevertheless, in our study, the age at menopause onset did not correlate with sarcopenia. It seems that early menopause does not have a significant influence on sarcopenia, presumably because other factors have a higher impact. Mood disorders favored the occurrence of sarcopenia possibly due to their effects both on the level of exercise and on a proper diet (Fujita S et al., 2004). Post-menopausal women from rural areas have a lower prevalence of sarcopenia, most probably due to their lifestyle that includes a higher level of exercise and also possibly due to a longer exposure to sun, Vitamin D being involved in striated muscle metabolism. We could not evaluate the correlation with serum levels of Vitamin D. Level of school education does not seem to have any impact on muscular performance. However, the presence of a support network, especially living with a family member has a significant role in preventing sarcopenia. In addition, income level was negatively correlated with sarcopenia, a lower income being associated with increased risk of physical disability, possibly by having a negative impact on dietary habits. Increased risk of malnutrition was strongly associated with lower gait speeds. Higher income potentially linked to better quality nutrition represents a protective factor against the risk of sarcopenia in older women. Living in rural areas and having family support are beneficial circumstances reflected in a lower risk of physical disability. Larger, longitudinal studies are needed to identify lifestyle and socio-economic factors that have a potentially significant impact on sarcopenia in older age.

\section{Conclusion}

Factors such as the presence of mood disorders and enhanced risk of malnutrition have a significant influence on the occurrence of sarcopenia and they need a careful assessment and management in order to prevent the onset of this condition considered a precursor of frailty syndrome. Older women living alone, in urban areas and with low income are especially at risk for physical disability.

\section{References}

1. Bohannon, R.W., Williams Andrews, A., 2011. Normal walking speed: a descriptive metaanalysis. Physiotherapy, Sep, 97(3), pp.182-9.

2. Cawthon, P.M., Blackwell, T.L., Cauley, J., et al., 2015. An evaluation of the usefulness of consensus definitions of sarcopenia in older men: results from the observational Osteoporotic Fractures in Men (MrOS) cohort study. Journal of the American Geriatrics Society, 63(11), pp.2247-2259.

3. Clegg, A., Young, J., Iliffe, S., Rikkert, M.O., Rockwood, K., 2013. Frailty in elderly people. Lancet, Mar 2, 381 (9868), pp.752-762. 
4. Collamati, A., Marzetti, E., Calvani, R., Tosato, M., D’Angelo, E., Sisto, A. N., \& Landi, F., 2016. Sarcopenia in heart failure: mechanisms and therapeutic strategies. Journal of Geriatric Cardiology : JGC, 13(7), pp.615-624.

5. Cruz-Jentoft, A.J., Baeyens, J.P., Bauer, J.M., Boirie, Y., Cederholm, T., Landi, Fr., Martin, F.C., Michel, J. -P., Rolland, Y., Schneider, S.M., Topinková, E., Vandewoude, M., Zamboni, M., 2010. Sarcopenia: European consensus on definition and diagnosis Report of the European Working Group on Sarcopenia in Older People. Age Ageing, Jul, 39(4), pp.412-423.

6. Cruz-Jentoft, A.J., Landi, F., Schneider, S.M., Zuniga, C., 2014. Prevalence of and interventions for sarcopenia in ageing adults: a systematic review. Report of the International Sarcopenia Initiative (EWGSOP and IWGS). Age and Ageing, 43, pp.748-759.

7. Diz, B.M.J., Zille de Queiroz, B., Tavares, L.B., Pereira, L.S.M., 2015. Prevalence of sarcopenia among the elderly: findings from broad cross-sectional studies in a range of countries. Rev Bras Geratr Gerontol, Rio de Janeiro, 18(3), pp.665-678.

8. Dodds, R.M., Roberts, H.C., Cooper, C., Sayer, A.A., 2015. The epidemiology of sarcopenia. $J$. Clin. Densitom, Oct-Dec, 18(4), pp.461-466.

9. Dodds, R.M., Sayer, A.A., 2016. Sarcopenia and frailty: new challenges for clinical practice. Clinical Medicine, 16(5), pp.455- 458.

10. Fujita, S. and Volpi, E., 2004. Nutrition and sarcopenia of ageing. Nutrition Research Reviews, 17(1), pp.69-76.

11. Guigoz, Y., 2006. The Mini-Nutritional Assessment (MNA®) Review of the Literature - What does it tell us? J Nutr Health Aging, 10, pp.466-487.

12. Kaiser, M., Bandinelli, S., Lunenfeld, B., 2010. Frailty and the role of nutrition in older people. A review of the current literature. Acta Biomedica, 81, Suppl 1, pp.37-45.

13. Lindle, R.S., Metter, E.J., Lynch, N.A., Fleg, J.L., Fozard, J.L., Tobin, J., et al., 1997. Age and gender comparisons of muscle strength in 654 women and men aged 20-93 yr. $J$ Appl Physiol, 83, pp.1581-7.

14. Maltais, M.L., Desroches, J., Dionne, I.J., 2009. Changes in muscle mass and strength after menopause. J Musculoskelet Neuronal Interact, Oct-Dec, 9(4), pp.186-97.

15. McGinn, A.P., Kaplan, R.C., Verghese, J., Rosenbaum, D.M., Psaty, B.M., Baird, A.E., Lynch, J.K., Wolf, P.A., Kooperberg, C., Larson, J.C., Wassertheil-Smoller, S., 2008. Walking Speed and Risk of Incident Ischemic Stroke Among Postmenopausal Women. Stroke, 39, pp.12331239.

16. Messier, V., Rabasa-Lhoret, R., Barbat-Artigas, S., Elisha, B., Karelis, A.D., Aubertin-Leheudre, M., 2011. Menopause and sarcopenia: A potential role for sex hormones. Maturitas., Apr, 68(4), pp.331-6.

17. Millan, A.C., Potter, J., Vivanti, A., Avenell, A., 2009. Protein and energy supplementation in elderly people at risk from malnutrition. Cochrane Database Systematic review, Apr 15;(2): CD003288

18. Park, H.M., Kim, T., 2013. Prevalence of sarcopenia and its association with osteoporosis in Korean postmenopausal women. Bone Abstracts, 1, p.297.

19. Rubenstein, L.Z., Harker, J.O., Salva, A., Guigoz, Y., Vellas, B., 2001. Screening for Undernutrition in Geriatric Practice: Developing the Short-Form Mini Nutritional Assessment (MNA-SF). J. Geront, 56A: M366-377.

20. Santilli, V., Bernetti, A., Mangone, M., Paoloni, M., 2014. Clinical definition of sarcopenia. Clinical Cases in Mineral and Bone Metabolism, 11(3), pp.177-180. 